\title{
Delone sets and Riesz basis
}

\author{
By Hideo SUZUKI \\ Graduate School of Mathematics, Kyushu University \\ (Communicated by Kiyosi ITÔ, M. J. A., Jan. 12, 1998)
}

\begin{abstract}
In this paper we deal with the density of Delone set and apply it constructing Riesz basis for an Hilbert space.
\end{abstract}

1. Introduction. A Riesz basis for Hilbert space is easily constructed by exponential maps over a periodic set. This drives us to the question how it is when a periodic set is replaces by Delone set. Construction by exponential functions will certainly work if a Delone set is very close to a periodic set. We are concerned with the problem how a Delone set can be different from the periodic set. In fact, Kadec and Levinson studied such a problem in the case of $L^{p}[-\pi, \pi]$ ( $p$ is a natural number) (see [6] pp. 118-131).

The purpose of the present note is to explore a little further in the cases of $L^{2}[-\pi$, $\pi]$ and $H^{1}[-\pi, \pi]$ (see our main theorem 5.3 and 5.4 below [6]).

\section{Delone set and Voronoi cell.}

Definition 2.1. An $(R, r)$-Delone set $\Lambda \subset$ $R^{N}$ is defined by the next two conditions (see [5] p. 28).

1) Discretness: There exists a positive real number $r$ such that for every $x, y \in \Lambda,|x-y| \geq$ $2 r$.

2) Relative density: There is a positive real number $R$ such that every sphere of radius greater than $R$ contains at least one point of $\Lambda$ in its interior.

Definition 2.2. Let $\Lambda \subset R^{N}$ be any Delone set. The Voronoi cell at a point $x \in \Lambda$ is the set of points of $R^{N}$ that lie at least as close to $x$ as to any other point of $\Lambda$ :

$$
V(x)=\left\{u \in R^{N} \| x-y|\leq| y-u \mid, y \in \Lambda\right\} .
$$

The Voronoi cell $V(x)$ is then the smallest convex region about $x$ (see [5] p. 42). gruent.

If $\Lambda$ is a lattice, the Voronoi cells are con-

Here we deal with a Delone set $\Lambda$ including $0: 0 \in \Lambda$.

3. The density of Delone set. We introduce the notion of the density for the $(R, r)$-Delone set. The density $\Delta$ of Delone set $\Lambda$ centered at $x$ is defined by

$$
\Delta_{x}(\Lambda)=\lim _{s \rightarrow \infty} \frac{\#\left\{\Lambda \cap B_{x}(s)\right\}}{m\left(B_{x}(s)\right)}
$$

( $m$ is the Lebesgue measure).

If (1) is well-defined, we say that $\Lambda$ has the density $\Delta_{x}(\Lambda)$ at $x$.

Here, we should notice that $\Delta_{x}(\Lambda)$ is actually independent of $x \in R^{N}$.

Lemma 3.1.

$$
\Delta_{x}(\Lambda)=\Delta_{0}(\Lambda)
$$

for all $x$. $R^{N}$.

Proof. Let $\Delta_{x}(\Lambda)$ be defined for a fixed $x \in$

Take $s>0$ such that $s>|x|$. Then

$$
B_{x}(s-|x|) \subset B_{0}(s) \subset B_{x}(s+|x|) .
$$

Here $|x|=\sqrt{x_{1}{ }^{2}+x_{2}{ }^{2}+\cdots+x_{N}{ }^{2}}$ for $x=\left(x_{1}\right.$, $\left.x_{2}, \ldots, x_{N}\right)$.

Therefore

$$
B_{x}(s-|x|) \cap \Lambda \subset B_{0}(s) \cap \Lambda \subset B_{x}(s+|x|) \cap \Lambda \text {. }
$$

We obtain

$$
\begin{gathered}
\frac{\#\left\{B_{x}(s-|x|) \cap \Lambda\right\}}{m\left(B_{0}(s)\right)} \leq \frac{\#\left\{B_{0}(s) \cap \Lambda\right\}}{m\left(B_{0}(s)\right)} \\
\leq \frac{\#\left\{B_{x}(s+|x|) \cap \Lambda\right\}}{m\left(B_{0}(s)\right)} . \\
\frac{\#\left\{B_{x}(s-|x|) \cap \Lambda\right\}}{m\left(B_{0}(s-|x|)\right)}\left\{\frac{s-|x|}{s}\right\}^{N} \leq \frac{\#\left\{B_{0}(s) \cap \Lambda\right\}}{m\left(B_{0}(s)\right)} \\
\leq\left(\frac{\#\left\{B_{x}(s+|x|) \cap \Lambda\right\}}{m\left(B_{0}(s+|x|)\right)}\right)\left\{\frac{s+|x|}{s}\right\}^{N}
\end{gathered}
$$

(\# is the number of elements).

We have (2) when $s \rightarrow \infty$.

Corollary 3.2.

for $x \neq y$.

$$
\Delta_{x}(\Lambda)=\Delta_{y}(\Lambda),
$$

We now define the density of $\Lambda$ by

$$
\Delta(\Lambda)=\lim _{s \rightarrow \infty} \frac{\#\left\{\Lambda \cap B_{0}(s)\right\}}{m\left(B_{0}(s)\right)}
$$

and call $\Delta(\Lambda)$ the density of $\Lambda$. 
For a $(R, r)$-Delone set, (minimal volume of Voronoi cells) $\leq \frac{1}{\Delta(\Lambda)} \leq$ (maximal volume of Voronoi cells).

Thus,

$$
O\left\{\frac{1}{R^{N}}\right\} \leq \Delta(\Lambda) \leq O\left\{\frac{1}{r^{N}}\right\} .
$$

If $\Lambda$ is a lattice, Landau showed the following results [1], [2], and [3].

\section{Theorem 3.3.}

$$
\Delta\left(Z^{N}\right)=1 .
$$

Theorem 3.4. Let $A$ be a regular $N \times N$ real matrix. then

$$
\Delta\left(A\left(Z^{N}\right)\right)=|\operatorname{det} A|^{-1} .
$$

That is, $\frac{1}{\Delta(\Lambda)}$ is the volume of each Voronoi cell in $\Lambda$.

4. Special Delone set. Definition 4.1. Let $\Lambda$ be a set including 0 . We say $\Lambda$ is an $L$-special set $(L<1 / 4)$ if there exists a regular matrix $A$ such that

a) \#( $\left.D_{L}(n) \cap A^{-1}(\Lambda)\right)=1$ for any $n \in Z^{N}$.

b) $A^{-1}(\Lambda) \subset \cup_{n \in Z^{N}} D_{L}(n)$.

Here $D_{L} \quad(n)=\left\{x \in R^{N}|| x_{j}-n_{j} \mid<L ; 1 \leq j\right.$ $\leq N\}$ for $n=\left(n_{1}, \ldots, n_{N}\right)$.

$A$ is called a lattice matrix and $\Lambda^{\prime}=A\left(Z^{N}\right)$ the periodic lattice associated with $\Lambda$.

Lemma 4.2. If $\Lambda$ is an $L$-special set, it is a Delone set. We freshly call $\Lambda L$-special Delone set. $\Lambda^{\prime}$ is not unique, but we see.

Lemma 4.3. Let $\Lambda^{*}$ be an $L$-special Delone set. Then,

$$
\Delta(\Lambda)=\Delta\left(\Lambda^{\prime}\right)=|\operatorname{det} A|^{-1} .
$$

$\Lambda^{\prime}$ is the periodic lattice associated with $\Lambda$.

Proof. (5) is a consequence of Theorem 3.4 and the next relation:

$$
\begin{aligned}
\#\left(\Lambda \cap B_{0}(s-2 R)\right) & \leq \#\left(\Lambda^{\prime} \cap B_{0}(s)\right) \\
& \leq \#\left(\Lambda \cap B_{0}(s+2 R)\right)
\end{aligned}
$$

valid for all $s>2 R$.

Then, we have (5).

5. Delone set and Riesz basis. Recall that a basis $\left\{r_{n}\right\}$ of a Hilbert space $X$ is a Riesz basis if there is a bounded invertible operator $T$ and an orthonormal basis $\left\{b_{n}\right\}$ in $X$ such that $r_{n}=T b_{n}$ for all $n$ ([6] p. 31).

Theorem 5.1 (Kadec's $1 / 4$ theorem). Let $\left\{\lambda_{n}\right\} \quad$ satisfy $\sup \left|\lambda_{n}-n\right|<L<1 / 4$. Then $\left\{\exp i \lambda_{n} t\right\}$ is a Riesz basis for $L^{2}[-\pi, \pi]$ (see [6] p. 42).

Lemma 5.2. Let $\left\{\boldsymbol{e}_{n}\right\}$ be an orthonormal basis for a Hilbert space $H$. Suppose $\left\{f_{n}\right\} \subset H$ be "close enough" to $\left\{e_{n}\right\}$ in the sense that

$$
\left\|\sum c_{k}\left(e_{k}-f_{k}\right)\right\|_{2} \leq \mu \sqrt{\sum\left|c_{k}\right|^{2}},
$$

for some constant $\mu ; 0 \leq \mu<1$, and arbitrary scalars $\left\{c_{n}\right\}\left(\|\cdot\|_{2}\right.$ is the $L^{2}[-\pi, \pi]$-norm).

Then $\left\{f_{n}\right\}$ is a Riesz basis for $L^{2}[-\pi$, $\pi$ ] (see [6] p. 40).

Our first main result reads as follows.

Theorem 5.3. Let $\Lambda$ be an $L$-special Delone set associated with a periodic lattice $\Lambda^{\prime}=$ $A\left(Z^{N}\right)$ on $R^{N}$.

If $\Lambda(\Delta)$ satisfy a) or b):

$$
\begin{aligned}
& \text { a) } \Delta(\Lambda) \leq \frac{1}{\left(2^{N}-1\right)^{2}} \\
& \text { b) } \Delta(\Lambda)>\frac{1}{\left(2^{N}-1\right)^{2}} \text { and } \\
& L<\frac{1}{4}-\frac{1}{\pi} \sin ^{-1} \frac{2-\sqrt[N]{1+\Delta(\Lambda)^{-\frac{1}{2}}}}{\sqrt{2}},
\end{aligned}
$$

then $\{\exp (i \lambda \cdot x)\}_{\lambda \in A}$ forms a Riesz basis for $L^{2}$ $\left(W_{A}(0)\right)$. Here $\left.W_{A}(0)=(2 \pi)^{T} A^{-1} V(0)\right)$ for the Voronoi cell $V(0)$ at $0 \in Z^{N}$, and $\lambda \cdot x$ is the inner product of $\lambda, x \in R^{N}$.

Proof. We denote the unique element $\lambda \in\{A$ $(V(0))+A k\} \cap \Lambda$ by $\lambda_{k}, k \in Z^{N}$.

Since $\left\{\exp \left(i \lambda^{\prime} \cdot x\right)\right\}_{\lambda^{\prime} \in \Lambda^{\prime}}$ forms an orthonor mal basis for $L^{2}\left(W_{A}(0)\right)$, we have to show by Lemma 5.2 that

$$
\left\|\sum_{k \in Z^{N}} c_{k}\left(\exp \left(i \lambda_{k} \cdot x\right)-\exp (i A k \cdot x)\right)\right\|_{L^{2}\left(W_{A}(0)\right)}
$$

$<1$ whenever $\sum\left|c_{k}\right|^{2} \leq 1$.

Since $A^{-1} \lambda_{k} \in D_{L}(k)$, we set by the triangle inequality and Theorem 5.1,

$$
\begin{aligned}
& \left\|\sum_{k \in Z^{N}} c_{k}\left(\exp \left(i \lambda_{k} \cdot x\right)-\exp (i A k \cdot x)\right)\right\|_{L^{2}\left(W_{A}(0)\right)} \\
\leq & \left.\operatorname{det} A\right|^{-\frac{1}{2}}\left\|\sum_{k \in Z^{N}} c_{k}\left(\exp \left(i A^{-1} \lambda_{k} \cdot y\right)-\exp (i k \cdot y)\right)\right\|_{L^{2}(V(0))} \\
\leq & |\operatorname{det} A|^{-\frac{1}{2}}\left\{(2-\cos \pi L+\sin \pi L)^{N}-1\right\} .
\end{aligned}
$$

(7) implies that the right hand of (8) is smaller than 1.

Our second main result reads as follows:

Theorem 5.4. Let $\Lambda$ be an $L$-special Delone set associated with $Z$. If

(9) $2(1-\cos \pi L+\sin \pi L)^{2}+8 L^{2}<1$, then $\left\{\frac{\exp \text { iat }}{\sqrt{a^{2}+1}}\right\}_{a \in A}$ is a Riesz basis for $H^{1}[-\pi$, $\pi]$. Proof. As $\left\{\frac{\exp i k x}{\sqrt{k^{2}+1}}\right\}_{k=-\infty}^{\infty}$ forms an ortho- 
normal basis for $H^{1}[-\pi, \pi]$, we have to show by Lemma 5.2 that

$$
\left\|\sum c_{k}\left(\frac{\exp i a_{k} x}{\sqrt{a_{k}^{2}+1}}-\frac{\exp i k x}{\sqrt{k^{2}+1}}\right)\right\|_{H^{1}}^{2}<1
$$

whenever $\sum\left|c_{k}\right|^{2} \leq 1 \quad\left(\|\cdot\|_{H^{1}}\right.$ is the $H^{1}[-\pi$, $\pi]$-norm).

$$
\begin{aligned}
& \left\|\sum c_{k}\left\{\frac{\exp i a_{k} x}{\sqrt{a_{k}^{2}+1}}-\frac{\exp i k x}{\sqrt{k^{2}+1}}\right\}\right\|_{H^{1}}^{2} \\
\leq & 2\left\|\sum \frac{c_{k}}{\sqrt{a_{k}^{2}+1}}\left(\exp i a_{k} x-\exp i k x\right)\right\|_{2}^{2} \\
+ & 2\left\|\sum c_{k}\left\{\frac{1}{\sqrt{a_{k}^{2}+1}}-\frac{1}{\sqrt{k^{2}+1}}\right\} \exp i k x\right\|_{2}^{2} \\
+ & 2\left\|\sum\left\{\frac{c_{k} a_{k}}{\sqrt{a_{k}^{2}+1}}\right\}\left(\exp i a_{k} x-\exp i k x\right)\right\|_{2}^{2} \\
+ & 4\left\|\sum\left\{\frac{c_{k}\left(a_{k}-k\right)}{\sqrt{a_{k}^{2}+1}} \exp i k x\right\}\right\|_{2}^{2} \\
+ & 4\left\|\sum c_{k} k\left\{\frac{1}{\sqrt{a_{k}^{2}+1}}-\frac{1}{\sqrt{k^{2}+1}}\right\} \exp i k x\right\|_{2}^{2} .
\end{aligned}
$$

Recall that if $\sup \left|a_{k}-k\right| \leq L<1 / 4$, $\left\|\sum c_{k}\left(\exp i a_{k} x-\exp i k x\right)\right\|_{2}^{2}$

$$
<(1-\cos \pi L+\sin \pi L)^{2}<1
$$

for $\sum\left|c_{k}\right|^{2}<1$. Note also

$$
\begin{aligned}
& \sum\left|\frac{c_{k}}{\sqrt{a_{k}^{2}+1}}\right|^{2} \leq \sum\left|c_{k}\right|^{2}<1, \\
& \sum\left|\frac{c_{k} a_{k}}{\sqrt{a_{k}{ }^{2}+1}}\right|^{2} \leq \sum\left|c_{k}\right|^{2}<1,
\end{aligned}
$$

(10)

$$
\begin{aligned}
& \left\|\sum \frac{c_{k}}{\sqrt{a_{k}{ }^{2}+1}}\left(\exp i a_{k} x-\exp i k x\right)\right\|_{2}^{2} \\
+ & \left\|\sum \frac{c_{k} a_{k}}{\sqrt{a_{k}{ }^{2}+1}}\left(\exp i a_{k} x-\exp i k x\right)\right\|_{2}^{2} \\
\leq & (1-\cos \pi L+\sin \pi L)^{2} \sum \frac{\left|c_{k}\right|^{2}}{a_{k}{ }^{2}+1} \\
+ & (1-\cos \pi L+\sin \pi L)^{2} \sum \frac{a_{k}{ }^{2}\left|c_{k}\right|^{2}}{a_{k}{ }^{2}+1} \\
\leq & (1-\cos \pi L+\sin \pi L)^{2} \sum\left|c_{k}\right|^{2} \\
< & (1-\cos \pi L+\sin \pi L)^{2} .
\end{aligned}
$$

On the other hand,

$$
\begin{aligned}
& \left\|\sum c_{k}\left\{\frac{1}{\sqrt{a_{k}^{2}+1}}-\frac{1}{\sqrt{k^{2}+1}}\right\} \exp i k x\right\|_{2}^{2} \\
& +2\left\|\sum \frac{c_{k}\left(a_{k}-k\right)}{\sqrt{a_{k}{ }^{2}+1}} \exp i k x\right\|_{2}^{2}
\end{aligned}
$$

$$
\begin{aligned}
& +2\left\|\sum c_{k} k\left\{\frac{1}{\sqrt{a_{k}^{2}+1}}-\frac{1}{\sqrt{k^{2}+1}}\right\} \exp i k x\right\|_{2}^{2} \\
& \leq \sum\left|c_{k}\right|^{2}\left\{\frac{\sqrt{k^{2}+1}-\sqrt{a_{k}^{2}+1}}{\left.\sqrt{a_{k}^{2}+1} \sqrt{k^{2}+1}\right\}^{2}}\right. \\
& +2 \sum\left|k c_{k}\right|^{2}\left\{\frac{\sqrt{k^{2}+1}-\sqrt{a_{k}^{2}+1}}{\sqrt{a_{k}^{2}+1} \sqrt{k^{2}+1}}\right\}^{2} \\
& +2 \sum\left|c_{k}\right|^{2}\left\{\frac{a_{k}-k}{\left.\sqrt{k^{2}+1}\right\}^{2}}\right. \\
& \leq \sum\left|c_{k}\right|^{2}\left\{\frac{\left(2 k^{2}+1\right)\left(\sqrt{k^{2}+1}-\sqrt{\left.a_{k}^{2}+1\right)}\right.}{\sqrt{\left(k^{2}+1\right)\left(a_{k}^{2}+1\right)}}\right\}^{2} \\
& +\sum\left|c_{k}\right|^{2}\left\{\frac{a_{k}-k}{\left.\sqrt{k^{2}+1}\right\}^{2}}\right. \\
& \leq \sum\left|c_{k}\right|^{2}\left\{\frac{4 k^{2}+3}{\left(k^{2}+1\right)\left(a_{k}^{2}+1\right)}\left(k-a_{k}\right)^{2}\right\} \\
& \leq 4 \sum\left|c_{k}\right|^{2}\left\{\frac{\left(a_{k}-k\right)^{2}}{a_{k}^{2}+1}\right\} \\
& \leq 4 L^{2} \sum \frac{\left|c_{k}\right|^{2}}{a_{k}^{2}+1} \\
& \leq 4 L^{2} . \\
& \leq(9),(10),{ }^{2} \\
&
\end{aligned}
$$

By using (9), (10), and (11),

$$
\begin{aligned}
& \left\|\sum c_{k}\left(\frac{\exp i a_{k} x}{\sqrt{a_{k}^{2}+1}}-\frac{\exp i n x}{\sqrt{k^{2}+1}}\right)\right\|_{H^{1}}^{2} \\
< & 2(1-\cos \pi L+\sin \pi L)^{2}+8 L^{2} \\
< & 1 .
\end{aligned}
$$

\section{References}

[1] E. Landau: Einfürung in die elementare und analytische Theorie der algebraischen Zahlen und der Ideale. Teubner 2nd Ed. (1927).

[2] E. Landau: Vorlesungen über Zahlentheorie II . Hirzel (1927).

[3] E. Landau: Zur analytischen Zahlentheorie der de finiten quadratischen Formen. S.-B. Preuss. Akad. Wiss, pp. 458-476 (1915).

[4] John J. Benedetto: Irregular Sampling and Frames. (eds. Wavelets-A Tutorial in Theory and Applications C. K. Chui). Academic Press Inc., pp. 445-507 (1992).

[ 5 ] M. Senechal: Quasicrystals and Geometry. Cambridge University Press, Cambridge (1995).

[6] R. M. Young: An Introduction to Nonharmonic Fourier Series. Academic Press Inc., New York (1980). 